\title{
Endovascular Recanalization in Acute Ischemic Stroke Using the Solitaire FR Revascularization Device with Adjunctive C-Arm CT Imaging
}

\author{
B.D. Mitchell, P. Chinnadurai, G. Chintalapani, H.A. Morsi, H. Shaltoni, and M.E. Mawad
}

\begin{abstract}
SUMMARY: In this clinical report, we examined a single-center experience by using the Solitaire FR Revascularization Device in the treatment of acute ischemic stroke in which there was poor initial visualization of the occluded arterial branches by using biplanar cerebral angiography. In all cases, adjunctive C-arm CT was used during the deployment of the thrombectomy device to gain additional information regarding device placement and expansion. Outcome measures included the extent of reperfusion, posttreatment changes in NIHSS scores, posttreatment $\mathrm{TICl}$ scores, cerebral hemorrhage, and survival. Clot removal with successful arterial recanalization was achieved in $15 / 18$ cases (83.3\%) with $\mathrm{TICl}$ scores of $2 \mathrm{~b} / 3$ in all patients who had initial recanalization. The NIHSS score improved, on average, from 19 pretreatment to 11 posttreatment, and $72 \%$ of patients survived. In cases of acute stroke in which there is little information available regarding the positioning and deployment of a retrievable stent during mechanical thrombectomy, the use of C-arm CT may provide more information about device placement across an area of thrombus.
\end{abstract}

l

cases of embolic stroke in which the suspected thrombus is large and causes total occlusion of a major cerebral vessel, such as the internal carotid, middle cerebral, or basilar artery, the endovascular navigation of the intracranial vessels can be challenging, with no contrast entering the vessels ahead of the catheters or interventional devices. Recently, the use of self-expanding retrievable stents (Solitaire FR Revascularization Device; Covidien, Irvine, California; Trevo; Stryker, Kalamazoo, Michigan) has complemented the current armamentarium of endovascular treatments available for acute stroke. ${ }^{1-5}$ Ideally, these stents are deployed within a suspected thrombus, and the stent is allowed to expand for a few minutes, at which point the stent can be retrieved, along with the thrombus.

The use of C-arm CT during endovascular interventional procedures has evolved rapidly from a technique that was relatively slow, with image quality lagging behind that of conventional CT, to one that now offers very high-resolution images that can be obtained rapidly during an interventional procedure. ${ }^{6}$ Recently, C-arm CT has been used for rapid acquisition of intraprocedural CT scans and ce-

Received July 14, 2014; accepted after revision November 18

From the Departments of Neurosurgery (B.D.M.) and Neuro-Interventional Radiology (H.A.M., H.S., M.E.M.), Baylor College of Medicine, Houston, Texas; Siemens Medical Solutions USA (P.C., G.C.), Angiography Division, Hoffman Estates, Illinois; and Neurovascular Center (H.A.M., H.S., M.E.M.), Baylor St. Luke's Hospital, Houston, Texas.

Please address correspondence to Bartley D. Mitchell, MD, PhD, Department of Neurosurgery, Baylor College of Medicine, 7200 Cambridge, Suite 9A, Houston, TX 77030; e-mail: bartley.mitchell@bcm.edu

http://dx.doi.org/10.3174/ajnr.A4256 rebral blood volume testing, especially during the treatment of patients with stroke, ${ }^{7,8}$ and can additionally provide high-quality images, even in areas of highly attenuated skull base anatomy.

Here, we report a series of 18 patients undergoing mechanical thrombectomy for acute stroke, in which initial visualization of the entire arterial anatomy was poor by using biplanar fluoroscopic methods, given the degree of occlusion (most frequently involving the entire internal carotid artery) from the suspected thrombus. In these cases, C-arm CT was used to aid overall visualization of the pertinent anatomy and to directly observe the degree of stent expansion in areas of suspected thrombus.

\section{MATERIALS AND METHODS}

Cases of 18 patients with acute stroke who underwent mechanical thrombectomy by using the Solitaire FR device at a single institution (Baylor College of Medicine) from October 2012 to January 2014 were retrospectively analyzed. Cases were selected on the basis of the following: 1) Acute stroke symptoms were present, evaluated by the neurology stroke team of the institution, with or without tPA given before angiographic evaluation. 2) Patients were angiographically evaluated within 8 hours of witnessed symptom onset. 3) There was no visualization of a major arterial branch (ICA, MCA, anterior cerebral artery, vertebral artery, or basilar artery) by using biplanar angiographic methods. 4) C-arm CT was used adjunctively for additional radiographic information during the treatment stage of the attempted thrombectomy. In each case in which the selection criteria were met, C-arm CT (syngo Dyna CT; Siemens, Erlangen, Germany) was used during 


\begin{tabular}{|c|c|c|c|c|c|c|c|c|}
\hline Patient No. & Age (yr) & Sex & Thrombus Location & No. of Passes & TICI Score (Post) & NIHSS (Pre) & NIHSS (Post) & Outcome \\
\hline 1 & 66 & $\mathrm{~F}$ & Left ICA & 1 & $2 b$ & 26 & 26 & Died \\
\hline 2 & 74 & $M$ & Right MCA & 2 & 0 & 17 & 17 & Died \\
\hline 3 & 67 & $M$ & Left MCA & 2 & $2 b$ & 23 & 9 & Rehab/Home \\
\hline 4 & 76 & $\mathrm{~F}$ & Right MCA & 1 & 3 & 15 & 15 & Rehab/Home \\
\hline 5 & 65 & $M$ & Right ICA & 2 & $2 b$ & 16 & 12 & Rehab/Home \\
\hline 6 & 67 & $M$ & Right MCA & 1 & 3 & 18 & 18 & Died \\
\hline 7 & 58 & $M$ & Right ICA & 2 & 3 & 18 & 2 & Home \\
\hline 8 & 58 & $M$ & Left MCA & 1 & $2 b$ & 27 & 11 & Rehab/Home \\
\hline 9 & 71 & $M$ & Left MCA & 2 & $2 b$ & 18 & 5 & Rehab/Home \\
\hline 10 & 65 & $M$ & Left MCA & 1 & $2 b$ & 16 & 2 & Rehab/Home \\
\hline 11 & 71 & $M$ & Left MCA & 2 & 3 & 19 & 19 & Died \\
\hline 12 & 60 & $M$ & Right MCA & 1 & 3 & 8 & 0 & Home \\
\hline 13 & 67 & $\mathrm{~F}$ & Left MCA & 1 & 3 & 15 & 1 & Home \\
\hline 14 & 77 & $M$ & Left MCA & 2 & 0 & 21 & 18 & LTAC \\
\hline 15 & 27 & $M$ & Left ICA & 1 & 3 & 22 & 5 & Rehab/Home \\
\hline 16 & 79 & $\mathrm{~F}$ & Left MCA & 4 & 0 & 24 & 24 & Died \\
\hline 17 & 30 & $M$ & Right MCA & 3 & $2 b$ & 18 & 7 & Rehab/Home \\
\hline 18 & 60 & $M$ & Right MCA & 2 & $2 b$ & 14 & 7 & Rehab/Home \\
\hline
\end{tabular}

Note:-Pre indicates before; Post, after; Rehab, rehabilitation; LTAC, long-term acute care facility.

stent deployment at both the initial time of deployment and following a 5-minute period in which the stent was allowed to expand into the presumed clot.

Intraprocedural C-arm CT images (20-second protocol; effective dose, $2.9 \mathrm{mSv}$ ) were acquired in 18 patients who underwent endovascular treatment for acute ischemic stroke. C-arm CT images acquired during mechanical thrombectomy passes were coregistered together (syngo InSpace 3D Fusion; Siemens) to understand and compare the stent configuration.

Following the 5-minute waiting period to allow the stent to expand into the suspected thrombus, the stent was retracted, while using an inflatable balloon catheter and with syringe suction applied to the guide catheter to prevent any loss of the thrombus during retrieval. Additional C-arm CT images of the retrievable stents were not obtained in each case in which additional stent passes were used beyond the first pass, unless there was evidence of arterial occlusion in a separate region that was not readily visualized with fluoroscopic methods. Selective angiography was performed between each pass of the Stentriever device (Trevo) to determine the extent of recanalization from the intervention and to determine whether another pass might be of benefit in cases in which incomplete recanalization was observed.

This study was performed with human institutional review board approval: collaborative protocol \#H-33379.

\section{RESULTS}

In this clinical report of 18 patients with acute stroke treated with retrievable self-expanding stents for mechanical thrombectomy, initial visualization of occluded arterial anatomy was poor, and in these cases, C-arm CT was used to obtain 3D images of the stent devices at the time of deployment, allowing improved visualization of stent position, shape, and conformation, compared with initially obtained digital subtraction angiography alone. In all cases in this series, there was an initial observable incomplete stent expansion in the presumed area of the arterial thrombus that could be seen with images obtained by using C-arm CT. In most cases (17/18 patients, $94 \%$ ), the initial incomplete stent expansion resolved after the initial 5-minute waiting period, indicating that the stent had expanded into the thrombus and was optimized for removal. In a single case, the stent did not show re-expansion, indicating the likely presence of an atherosclerotic embolus, as opposed to a softer thrombotic embolus. Overall, we were able to achieve a recanalization rate of $83.3 \%$ (15/18 patients) by using mechanical thrombectomy with retrievable stents alone. An average of 1.7 passes of the stent was used overall to achieve recanalization, with a maximum of 4 passes used in 1 case. A summary of the patient data is provided in the Table.

The NIHSS score was assessed immediately before the thrombectomy procedure and after the procedure in all cases, and overall, it was seen to improve from 19 (preprocedure) to 11 postthrombectomy ( $P=.001,2$-tailed $t$ test). If one considered only those patients who survived (13/18), their NIHSS score improved, on average, from 18 to 7 overall $(P<.0001,2$-tailed $t$ test $)$, and all surviving patients showed improved or stable NIHSS scores following treatment with the Solitaire device. Posttreatment hemorrhage was observed in $28 \%$ of patients (5/18), though the extent of hemorrhage was limited to petechial or small basal ganglia hemorrhage in all cases observed (grades: hemorrhagic transformation 1 in 3 cases and hemorrhagic transformation 2 in 2 cases). Despite the limited extent of hemorrhage, 4 of those 5 patients actually achieved recanalization during treatment and 2 of these 5 ultimately died; these results indicate that despite recanalization, patients may have poor outcomes.

\section{Case Illustration}

A 58-year-old man with a history of atrial fibrillation, on a direct thrombin inhibitor medication, presented with a witnessed sudden-onset unilateral weakness and collapse. He was not eligible for IV tPA, given his prior history of direct thrombin inhibitor use; therefore, he was taken to the angiography suite for definitive treatment. Initial angiography showed stagnation of blood flow into the right internal carotid artery, with no intracranial flow (Fig $1 A,-B)$, suggesting a likely $\mathrm{T}$-thrombus of the internal carotid artery bifurcation, extending onto both the proximal MCA and anterior cerebral artery, though this was presumed given the lack of visual data available from biplanar angiographic imaging alone. 


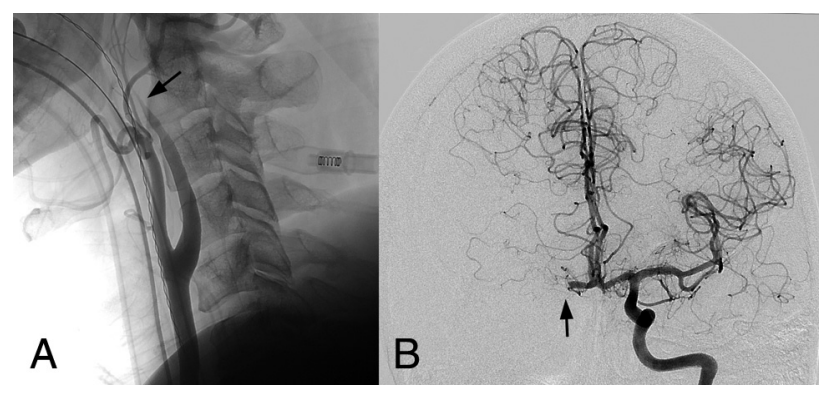

FIG 1. Preprocedural angiogram showing complete blockage of the right internal carotid artery. $A$, Oblique view of the carotid bifurcation shows complete ICA obstruction, with poor visualization of the vessels both proximal and distal to the presumed occlusion. B, Left ICA angiogram shows cross-filling perfusion into the right anterior cerebral artery territory, but with embolus blocking contralateral blood flow at the right anterior cerebral artery.

The right-sided anterior cerebral artery territory was cross-filled from the left carotid circulation via a patent anterior communicating artery, though the right MCA territory was completely devoid of circulation.

Further navigation of the guidewire and catheter into the right anterior circulation territory was severely limited, though the guidewire was able to be advanced into the suspected thrombus region. At this point, the Solitaire stent retrieval system was deployed within the proximal MCA, extending from the superior division of the MCA to the ICA bifurcation, though the extent of expansion of the stent was not easily viewed on conventional fluoroscopy (Fig 2A). C-arm CT with 3D reconstruction at this time showed that the Solitaire device was not fully expanded and was likely within the middle of the thrombus, unable to expand to the vessel inner walls (Fig 2B). Once retrieved, the stent was found to have removed significant amounts of fresh blood clot. The device was again deployed within the same area of the right MCA, though this time with the distal end in the inferior division of the MCA. C-arm CT during this second deployment showed significantly greater stent expansion to the margins of the vessel walls (Fig $2 C,-D$ ), though copious amounts of thrombus were again retrieved on this pass.

Following further aspiration of thrombi retained within the sheath, subsequent angiography demonstrated markedly improved blood perfusion into the right ICA/MCA/anterior cerebral artery territory, indicating a nearly complete return of blood flow to the area affected by the stroke (Fig 3). The patient recovered well, without weakness or paralysis, with no sensory loss and no speech difficulties, following successful removal of the large ICA bifurcation thrombus.

\section{DISCUSSION}

Catheter navigation and subsequent device deployment may be hindered during very large thrombus blockage of major arterial distributions during an acute stroke, especially in an ICA T-distribution thrombus, in which neither the MCA nor the anterior cerebral artery territories can be visualized. Effective treatment of this neurologically devastating condition depends largely on the rapid deployment of interventional stroke procedures, including retrievable stent devices. Although other stroke cases were treated at this institution during the time of this series, we only included patients who had large arterial segment occlusion with resulting



FIG 2. Comparison of images by using standard biplanar fluoroscopy versus C-arm CT. A, Anteroposterior fluoroscopic view of the deployed Solitaire stent device on the first pass across the thrombus. Visualization of the device is poor; arrows indicate approximate location of the stent. B, C-arm CT of the deployed stent across the thrombus. Note the narrow diameter of the stent, indicating the presence of surrounding thrombus, preventing the stent from full expansion. C, Anteroposterior fluoroscopic view of the second pass of the Solitaire device, again with poor visualization of the overall stent shape and conformation. D, C-arm CT of the second-pass of the Solitaire device. Now the stent is able to more fully expand within the artery, though there is still thrombus present. E, Retrieved thrombus from the first and second passes in the MCA.

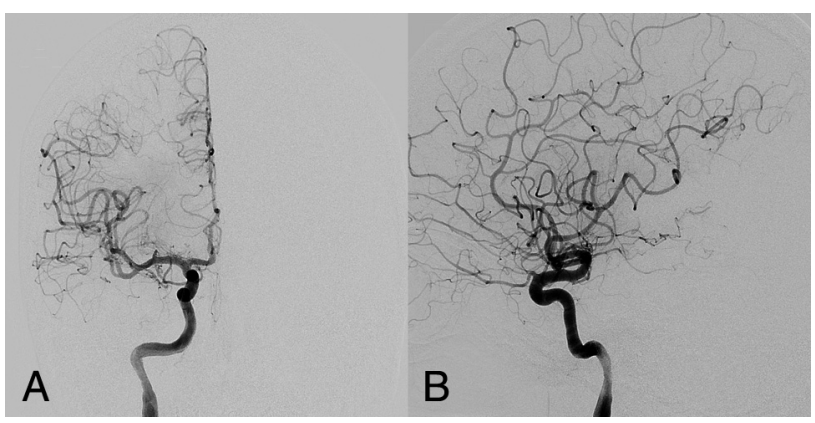

FIG 3. Postprocedural angiograms in anteroposterior $(A)$ and lateral (B) projections show complete recanalization of right MCA and anterior cerebral artery distributions following mechanical thrombectomy with combined C-arm CT and retrievable stent deployment.

decreased visualization during imaging. In such cases in which visualization of the arterial system is poor, the concurrent use of C-arm CT during the interventional procedure provides additional information to surmise whether one has deployed the thrombectomy device within the suspected area of the thrombus by observing the diameter of the stent on deployment. In the case 
of retrievable stents, the thrombus provides resistance for the stent to expand, giving it a stenotic appearance. Further comparison can also be made between the diameter of the deployed stent and the vessel diameter of the contralateral patent same-distribution arterial system. Whenever possible, however, before stent placement, we also analyze the contralateral arterial cerebral blood supply to determine whether there may be any underlying tortuous or difficult anatomy, such as congenital stenosis. We frequently use this method in our treatment center, especially in cases in which little or no information is available regarding the occluded vessel anatomy.

In this series, we showed a recanalization rate of $83 \%$, and in all cases in which recanalization was achieved, the posttreatment TICI score was either $2 \mathrm{~b}$ or 3 (near- or complete distal reperfusion). Our results with these cases of initial poor arterial visualization are comparable with those seen in the Solitaire With the Intention For Thrombectomy trial, with a recanalization rate of $83 \%$ in both studies ( $89 \%$ in the Solitaire With the Intention For Thrombectomy trial after rescue treatment). ${ }^{4,5}$ In all cases in this series the TICI scores were either zero or $2 b / 3$, indicating that in our hands this type of recanalization procedure was an all or nothing procedure: either achieving near- or complete reperfusion or no reperfusion at all, with no intermediate reperfusion results. Reperfusion, however, did not necessarily translate into good patient outcomes. Of the 5 patients in this series who ultimately died, 3 of them had a postprocedural TICI score of $2 b$ or 3 . Most interesting, 4 of the 6 patients who either died or had a very poor clinical outcome (incapable of self-care, requiring long-term acute care hospital placement) also did not receive tPA before the thrombectomy procedure for various reasons. Of the remaining patients who were able to go home, only 1 patient had not received tPA before thrombectomy.

In this series, we were able to show that in cases in which an arterial system cannot be adequately visualized initially by using standard biplanar angiographic methods, C-arm CT can provide additional information that may be useful in guiding clinical decision-making. In particular, this may allow visualization of the incomplete expansion of the thrombectomy device in the presumed region of the occlusive thrombus, where the occlusion would otherwise have been difficult or impossible to visualize, compared with standard fluoroscopic views alone. Stent retriever interaction with clot material has been shown to provide key information regarding the penetration of stent struts into the thrombus, ${ }^{9}$ and in this report, C-arm CT also allowed evaluation of the degree of stent expansion into the thrombotic region, further allowing the operator to determine whether the region of stenosis was from thrombus or possibly from a more firm embolus or an area of pre-existing stenosis from atherosclerosis or inherent vessel stenosis. This is critical information in the setting of acute treatment for arterial blockage because in the case of atherosclerotic, embolic, or inherent vessel stenosis, multiple attempts at recanalization would be futile and would perhaps even precipitate worse patient outcomes from stroke propagation, vessel damage, or cerebral hemorrhage.

The ability to preserve function following a stroke depends directly on the physician's ability to rapidly re-establish sufficient blood flow to the affected stroke area. Performing this task efficiently and reliably, with as much information as possible, is ideal and will likely lead to better patient outcomes, especially with large acute cerebral vessel occlusions as seen in these cases.

\section{CONCLUSIONS}

In the treatment of acute stroke when visualization of the occluded arterial branch is poor or impossible, the use of adjunctive $\mathrm{C}$-arm CT can provide additional information that can aid the operator in clinical decision-making regarding the adequacy of stent placement and thrombus position.

Disclosures: Ponraj Chinnadurai-RELATED: Employment: Siemens Medical Solutions USA Inc, Comments: full-time Staff Scientist, Angiography Division. Gouthami Chintalapani-RELATED: Employment: full-time employee at Siemens Medical Solutions USA Inc. Michel E. Mawad-RELATED: Support for Travel to Meetings for the Study or Other Purposes: Covidien, Comments: I traveled to give lectures on endovascular treatment of stroke and flow diversion in aneurysm treatment. Covidien paid my airfare, and I was paid a per diem for the time spent; UNRELATED: Payment for Lectures (including service on Speakers Bureaus) \& Travel/Accommodations/ Meeting Expenses Unrelated to Activities Listed: I traveled to give lectures on endovascular treatment of stroke and flow diversion in aneurysm treatment. Covidien paid my airfare and I was paid a per diem for the time spent.

\section{REFERENCES}

1. Dorn F, Stehle S, Lockau H, et al. Endovascular treatment of acute intracerebral artery occlusions with the Solitaire stent: single-centre experience with 108 recanalization procedures. Cerebrovasc Dis 2012;34:70-77

2. Koh JS, Lee SJ, Ryu CW, et al. Safety and efficacy of mechanical thrombectomy with Solitaire stent retrieval for acute ischemic stroke: a systematic review. Neurointervention 2012;7:1-9

3. Mendonça N, Flores A, Pagola J, et al. Trevo versus Solitaire: a headto-head comparison between two heavy weights of clot retrieval. J Neuroimaging 2014;24:167-70

4. Saver JL, Jahan R, Levy EI, et al. SOLITAIRE with the intention for thrombectomy (SWIFT) trial: design of a randomized, controlled, multicenter study comparing the SOLITAIRE flow restoration device and the MERCI retriever in acute ischaemic stroke. Int J Stroke 2014;9:658-68

5. Saver JL, Jahan R, Levy EI, et al. Solitaire flow restoration device versus the Merci retriever in patients with acute ischaemic stroke (SWIFT): a randomised, parallel-group, non-inferiority trial. Lancet 2012;380:1241-49

6. Kamran M, Nagaraja S, Byrne JV. C-arm flat detector computed tomography: the technique and its applications in interventional neuro-radiology. Neuroradiology 2010;52:319-27

7. Ganguly A, Fieselmann A, Boese J, et al. In vitro evaluation of the imaging accuracy of C-arm conebeam CT in cerebral perfusion imaging. Med Phys 2012;39:6652-59

8. Ganguly A, Fieselmann A, Marks M, et al. Cerebral CT perfusion using an interventional C-arm imaging system: cerebral blood flow measurements. AJNR Am J Neuroradiol 2011;32:1525-31

9. Wenger KJ, Berkefeld J, Wagner M. Flat panel detector computed tomography for the interaction between contrast-enhanced thrombi and stent retrievers in stroke therapy: a pilot study. Clin Neuroradiol 2014;24:251-54 